\title{
User-centred design in the development of a navigational aid for blind travellers
}

\author{
Helen Petrie ${ }^{1}$, Valerie Johnson ${ }^{1}$, Thomas Strothotte ${ }^{2}$, Rainer Michel ${ }^{2}$, \\ Andreas Raab ${ }^{2}$ and Lars Reichert ${ }^{3}$
}

\author{
1 Sensory Disabilities Research Unit, \\ University of Hertfordshire, \\ Hatfield AL10 9AB, U.K. \\ email: \{h.l.petrielv.johnson\}@ @erts.ac.uk \\ 2 Department of Simulation and Graphics, \\ Otto-von-Guericke University of Magdeburg, \\ Universitätsplatz 2, D-39106 Magdeburg, Germany \\ email: $\{$ tstr|raablrainer $\}$ isg.cs.uni-magdeburg.de \\ 3 Department of Computer Science, \\ Free University of Berlin, \\ Takustrasse 9, D-14195 Berlin, Germany \\ email: reichert@inf.fu-berlin.de
}

\begin{abstract}
Modern technology can assist disabled people in two ways: either technology can be used to design devices specifically to meet the needs of disabled people, or technology that is already used by able-bodied people can be adapted to become usable by disabled people. This paper presents an example of the former. The MoBIC (Mobility of Blind People Interacting with Computers) project is developing a new navigational aid which gives blind travellers navigational information based on the new technologies of geographical information systems (GIS) and the global positioning system (GPS) of satellites. The aid includes two components: a pre-journey planning system and a system which will assist travellers on journeys. The process by which initial prototypes for the system were developed is outlined and the elicitation of user requirements is discussed. Some results of initial evaluations conducted in the United Kingdom and the first field trials conducted in Germany are also presented.
\end{abstract}

KEY WORDS: visually disabled users, navigational aid, user-centred design, evaluation

Human-Computer Interaction: INTERACT'97 S. Howard, J. Hammond \& G. Lindgaard (editors)

Published by Chapman \& Hall OIFIP 1997 


\section{INTRODUCTION}

New technological innovations often fail to take account of the needs of elderly people and those with disabilities who find themselves at a disadvantage in many areas of life. For example, the facility to draw cash from an Automatic Teller Machine (ATM) is one many of us appreciate, but for those who use wheelchairs and cannot reach the card slot and those with impaired vision who cannot see the card slot or the instructions on the screen, the benefits of this facility are not so readily apparent. Increasingly, the benefits of new technology rely on the ability to interact visually i.e read a screen or use a mouse, and ATMs are just one example. Other examples include screen telephones and GUI-based computer applications.

Nonetheless, new technologies can be used to great advantage by people with disabilities and elderly people. Two ways in which technology can help these groups can be distinguished. Firstly, one can make mainstream technology accessible to a wider range of users, with differing needs and abilities. This will be referred to as "adaptive technology", as it adapts mainstream technology for use by a broader range of users (McMillan, 1992; Petrie, in press). The GUI problem mentioned above is an area in which this approach has been applied. Many people with visual or physical disabilities need to use computer systems and applications for employment or leisure. For example, people with visual disabilities have traditionally worked as telephone operators and more recently have found computer programming an employment area in which their disability is irrelevant to their productivity. But software in both these areas, and many others, is rapidly moving to GUI-based platforms and this is jeopardizing the employment opportunities for disabled people. Numerous efforts have been made to adapt GUI-based platforms for use by disabled people (Mynatt and Weber, 1994; Petrie, Morley and Weber, 1995).

The second way in which technology can help disabled and elderly people is to use technology to enable disabled and elderly people to perform tasks which they are currently unable to do or find extremely difficult. This domain of technological innovation will be referred to as "assistive technology", as disabled and elderly people are assisted in undertaking particular tasks by the technology. In this area, the ways these technologies are used may be completely different from the original intention, but assistive technology can use developments in many areas to greatly increase the quality of life of disabled and elderly people.
Two recent technological innovations are now available which allow the development of a new assistive technology for blind people. These technological innovations are computer-based geographical information systems (GIS) which give access to map information via a computer and the Global Positioning System (GPS) of satellites which provides information about the precise latitude and longitude of any position on the earth's surface, via a small receiver. These technologies are already being used in various navigational situations, for example to guide cars and planes.

The MoBIC (Mobility of Blind and elderly people Interacting with Computers) Project, funded by the European Union, is employing GPS and GIS technologies to guide blind travellers on a route which they can also plan on a home-based computer. This makes MoBIC an especially good example of assistive technology in that it is employing technologies already being used for other purposes to help disabled people to do something which was previously very difficult, if not impossible.

Whether one is designing for all the population, or only for a specific group with special requirements, it is important to include users in the design process to ensure that designers understand their requirements and capabilities. Much has been written about different methods for the involvement of users in designing interfaces to complex systems and the different methodologies which can be employed, such as usercentred design, participatory design and usability engineering (e.g. Nielsen, 1993; Norman and Draper, 1986; Schuler and Namioka, 1993; Whiteside, Bennett and Holtzblatt, 1988). These methodologies can also be applied to designing for disabled and elderly people, although a different set of problems and issues may arise.

This paper will outline initial designs for the two components of the MoBIC Travel Aid (MoTA). Some $\mathrm{HCI}$ techniques employed in the initial elicitation of user requirements which lead to the design of the MoTA aid and its progressive refinement will be discussed. Finally the evaluations and field trials of the first prototype implementations will be presented.

\section{COMPONENTS OF THE MOBIC TRAVEL AID}

The MoTA consists of two basic components: the MoBIC Pre-Journey System (MoPS) and the MoBIC Outdoor System (MoODS). 


\subsection{The MoBIC Pre-journey System}

The MoBIC Pre-journey System (MoPS) allows blind people to explore a map and plan their journey before setting out. These activities can be carried out using a standard PC computer with appropriate output (synthetic speech or Braille) and the specialized MoBIC software.

The heart of the MoPS is a database used to organize the information which is relevant to users' exploration and planning tasks. It contains such data as:

- map information obtained from public sources. This includes street names and building numbers, locations of pedestrian crossings and public buildings (for example post offices, libraries, police stations); and

- personal information, such as addresses and preferences (for example, avoiding uncontrolled street crossings or flights of steps).

In addition, there are data which are specific to the needs of blind travellers:

- notes on the traversability of certain streets and areas (for example, a particular street may have parking meters, trees or a great deal of clutter outside shops) and - information on specific surfaces underfoot (for example tactile tiles, cobbled pavements, grassed areas).

Users interact with MoPS to explore a map either using standard cursor keys or a touchtablet with a tactile overlay. To assist users in planning a particular route for a journey MoPS incorporates a route-calculation program which takes into consideration user preferences (e.g. use controlled crossings only; avoid steps; provide the shortest route to walk or by public transport). Figure 1 shows an example of the visual representation of digital map information. Such a presentation may be enlarged for those with partial vision and is also useful for sighted people assisting blind users.

\subsection{The MoBIC Outdoor System}

The MoBIC Outdoor System (MoODS) takes the journey plan developed with MoPS and assists travellers in reaching their destination by matching the information in its GIS against the traveller's position provided by a GPS receiver integrated into the MoODS system and a differential GPS signal which increases the accuracy of the GPS calculation. A Trip Management System advises the traveller by giving directions, warnings or other relevant information. The user interacts with the system by asking questions using a small, hand-held keypad and receiving answers verbally via an earphone which does not block other sounds from the environment. Special keys are reserved for questions such as "Where am I?" and "Where should I proceed from here?" It is important to note that the MoBIC system is primarily intended for the informational needs of travellers with respect to their overall journey. Most information about the immediate environment (e.g. an obstacle temporarily blocking the way) should still be provided by the long cane or guide dog.

\section{INITIAL DESIGN OF THE MoTA}

The first step in designing the MoTA was an extensive study of what blind people might find useful from such a system and how the information should be presented to users. A number of user requirements studies were conducted including in-depth interviews with both potential users of the system, mobility officers and others involved in the training and rehabilitation of blind people. Group discussions with potential users also took place. Finally, "Wizard of Oz" simulations of how the MoPS and MoODS components might function were made for potential users to try out to give them a concrete experience of what the system might be like and enable them to comment on different possibilities.

\subsection{User Requirements}

To understand the needs of blind and partially sighted people in relation to the MoBIC aid, a number of user requirements studies were undertaken within the MoBIC Project. Firstly, interviews were conducted with blind and partially sighted individuals concerning their current travel habits and problems which they encounter travelling in both familiar and unfamiliar environments, their ideas on what information the MoTA could provide and how that information should be presented. Secondly, interviews were conducted with teachers of mobility training and other individuals involved in this area, such as trainers of these teachers. Discussion groups were also held with blind, including elderly blind, people to elicit more general information about their views on the MoBIC aid. In addition further studies were conducted in which potential users were given mock-ups of aspects of the travel aid for assessment. A more detailed report of the elicitation of user requirements for the MoBIC Project can be found in Jansson (1995), and Petrie and Johnson (in press).

\section{2 "Wizard of Oz" study of the MoODS system}

Although the interviews and discussion groups yielded much useful information for the design of the MoTA, it was felt that many participants were having difficulty 


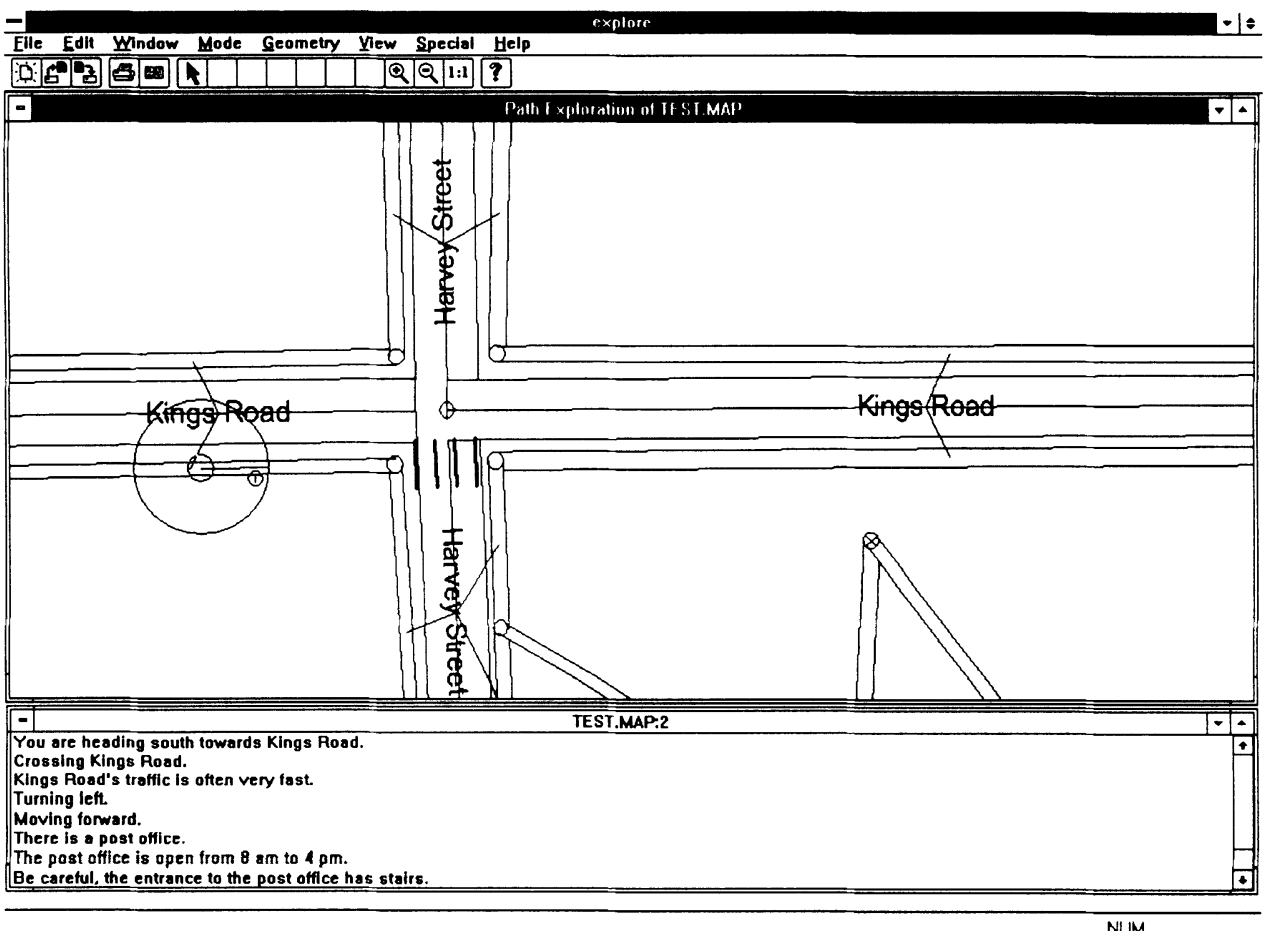

Figure 1: Example of the visual representation of digital map information

imagining what the systems might actually be like and how they could work. This is not surprising, as this is a completely new type of travel aid for blind people which no-one has had any experience with as yet. As an attempt to overcome this difficulty, a "Wizard of $\mathrm{Oz}$ " study of the MoODS system was conducted. Wizard of $\mathrm{Oz}$ studies are sometimes conducted in the design of complex computer systems (Landauer, 1987; Nielsen, 1993).

In the case of the MoODS system, when the study was conducted, work on the integration of the GPS and GIS components was still being undertaken, so a human being acted as the GPS receiver for the blind traveler. This was achieved in the following manner. Both authors walked a short route working out what instructions the MoODS system may give a traveler at different points on this route. These instructions were then recorded on a cassette tape with brief pauses in between each instruction. Then a number of blind people walked the route individually with their usual mobility aid, carrying a Walkman cassette player on which to listen to the instructions and accompanied by a sighted person (the second author). The pre-recorded instructions guided them from point to point along the route. At each point they paused and listened to the next instruction before proceeding. At the end of the route participants were asked a number of questions about their experience and asked to rate the system on a number of scales. It was stressed that what they were experiencing was only a simulation of part of the eventual MoODS system. This procedure allowed us to give potential users a more concrete idea of what using the MoODS system while travelling might be like and to see whether the nature of the speech messages given was appropriate (e.g. level of detail, format etc.)

Eighteen blind people took part in this study and all felt they had gained a much clearer idea of what MoBIC would be like and were able to give further suggestions for its design. They also gave positive feedback on the 
nature of the speech messages which was used to design the eventual format.

In order to find acceptable solutions to specific problems, both high and low fidelity prototypes of particular hardware components were presented to users. The interviews had identified a strong reluctance on the part of blind people to use earphones for receiving output from the MoODS and this extended to any device which might detract from the auditory cues on which those without sight depend so heavily. This problem was addressed as part of the Wizard of $\mathrm{Oz}$ study. Users evaluated three different earpiece and headset alternatives. The Sony Sports Headset MDR A17 (which has small earphones which can be placed in the ears or which sit in front of the ears, the latter configuration was used for this study), the Sony SR12 earpiece and the Sennheiser earpiece with earbow (both earpieces have a small earphone which sits in front of the ear). These were high fidelity prototypes in that they were fully functional and allowed users to experience genuine interaction. Participants rated alternatives on the following criteria:

Q1. How easy was it to hear the instructions?

( 1 = Very difficult -7 = Very easy)

Q2. How easy was it to use this device?

( 1 = Very difficult $-7=$ Very easy)

Q3. Did you feel at all conspicuous wearing it?

( $1=$ Very conspicuous $-7=$ Not at all conspicuous)

Q4. Did you feel the device masked useful

environmental sounds? ( $1=$ Greatly $-7=$ Not at all $)$

Q5. How happy would you be to use such a device?

( 1 = Definitely would not use -7 = Perfectly happy)

Q6. Overall rating of the device $(1=$ Very poor $-7=$ Excellent)

The mean ratings given are shown in Figure 2. Of the three headset and earpiece alternatives considered, the earpiece produced by Sennheiser was the most positively received. The Sony SR12 earpiece was not felt to have sufficient volume. However, participants indicated that could the volume be improved the earpiece would be quite an acceptable device. The Sony Sports Headset MDR A17 delivered information at an acceptable volume, but some people felt that wearing a headset of any kind would make them more conspicuous. The headset was also felt to be more awkward to use than an earpiece which simply clips round the ear.

In the case of input to the MoODS an initial prototype of a wrist worn keypad was presented to users along with several cardboard mock-ups representing a number of variations. Whilst users were not able to fully interact with these low fidelity prototypes they were able to judge what it would be like to wear them and how easy or difficult it would be to identify and operate the keys. Users also provided ratings on the usability of the prototype keypad as well as various features of the keys, including spacing, identification (i.e knowing which key is which), configuration and responsiveness (all rated on a 1 to 7 scale, with 7 being the most positive rating). These ratings are shown in Figure 3 below. These evaluations lead to a number of recommendations (see Table 1) which greatly influenced the final design of the MoODS keypad.

\section{INITIAL EVALUATIONS IN THE UNITED KINGDOM}

Throughout the MoBIC project components of the system were evaluated as they became available. An example is the evaluation of the use of cursor keys to navigate through a digital map when using the MoPS. Potential users had indicated that they felt they would be able to learn about an area in this way and such a simple solution would obviously be very cost-effective for blind people, as it would only require the purchase of a software component to use with their existing PC.

The evaluation was conducted at the University of Hertfordshire on and close to the Hatfield campus. Four blind people participated in this initial evaluation, two male and two female. Two were congenitally blind and two adventitiously blind. The term congenitally blind refers here to those who were born with some kind of visual impairment though they may have had some residual vision for the first few years of life. Two of the participants were guide dog users and two long cane users. Ages ranged from 29 to 45 years. Two routes were devised and employed in the evaluation which involved two phases. Although the routes were matched in terms of length and complexity the order of presentation was counterbalanced to control for any extraneous variables.

In Phase 1 of the evaluation the four participants were asked to familiarise themselves with a simulated route on a computer at the university. In Phase 2 participants were taken out to the actual route and asked to walk from the start point to the finish. At this stage there was no MoODS component available and a researcher undertook to provide the information which would ultimately be provided by the MoODS in another Wizard of $\mathrm{Oz}$ simulation. In addition to the recording of time, errors and requests for help in both phases, rating scales and open ended questions were administered to 


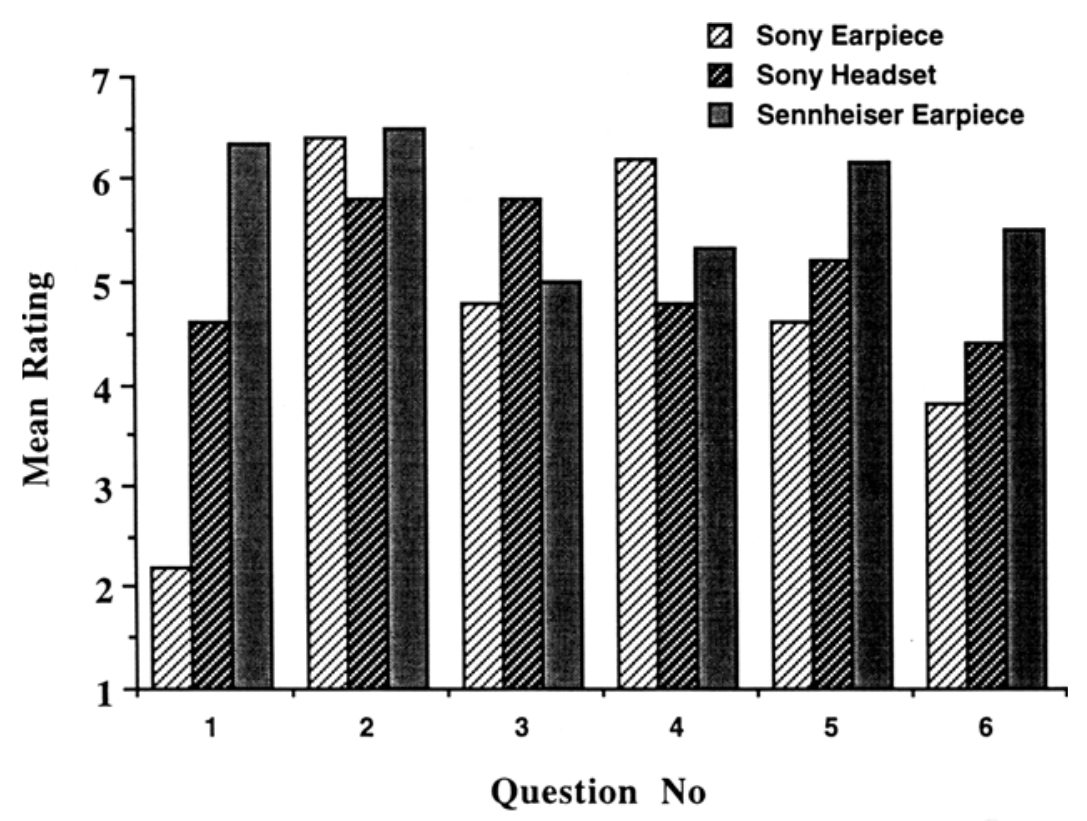

Figure 2: Mean usability ratings for headset/earpiece alternatives

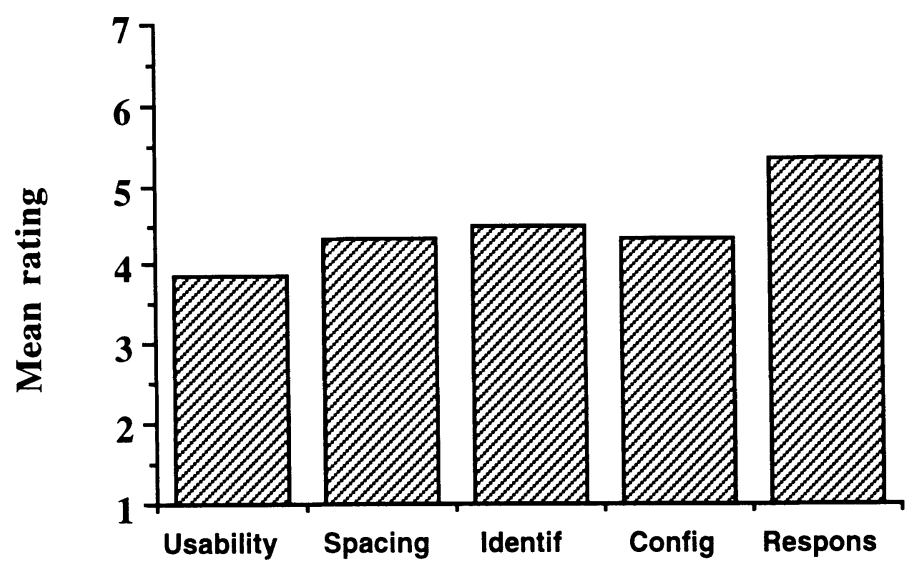

Figure 3: Mean ratings of features of the prototype keypad 
Table 1: Recommendations for overall features of keypad

\begin{tabular}{|l|l|}
\hline Feature & Recommendation \\
\hline Shape & Circular, provided the measurements below are not exceeded \\
\hline Diameter of keypad & No more than 6 centimetres (smaller if possible) \\
\hline Thickness of keypad & The keypad should be as thin as possible \\
\hline Connecting Cable & $\begin{array}{l}\text { This should be as thin as possible, preferably so that it will fit } \\
\text { inside the user's sleeve }\end{array}$ \\
\hline Wrist fastener & Wrist strap must fasten firmly but also needs to be adjustable. \\
\hline
\end{tabular}

assess usability and learnability of and satisfaction with the MoPS component.

No participant studied the route for longer than 30 minutes before going out to walk it and only one participant requested help when using the MoPS. There were no requests for help while walking the route and only one of the participants made one error in walking a route. Figure 4 shows that mean ratings of usability, learnability and satisfaction were all close to the top of the 7 point scale as were ratings of the usefulness of various types of information provided by the MoPS. Although all participants completed the route walk without difficulty and were very positive in their assessment of the MoTA this evaluation was also successful in identifying a number of areas in which the MoPS software could be improved and these improvements were implemented in subsequent versions of the system.

\section{FIELD TRIALS IN GERMANY}

The field trials in Germany were conducted at the Free University of Berlin. Six participants attended a total number of 25 tutorial sessions using prototype versions of both the MoPS and MoODS components. Four of the participants were blind and two were partially sighted, five were male and one female and ages ranged from 25 to 61 years. Onset of visual disabilities varied with some having problems from birth and some only recently. The trials lasted for three months and during this time the software was regularly updated to incorporate suggestions made by participants.

The general feedback from all participants at the end of the trial indicated a high satisfaction with the latest version of the MoPS software and provided encouraging comments on the MoODS component. Figure 4 shows that, as in Hatfield, mean ratings for the usefulness of various types of information were well above the midpoint on the scale for all types of information and that the lowest mean rating given was for distance information in both cases. Although this is still above the mid-point, distance information was considered more useful by some people than others because some find distance difficult to judge.

Figure 4 shows the mean ratings for usability and learnability of and satisfaction with the MoPS component when using cursor keys for navigation as given by participants in both the Hatfield evaluations and the Berlin field trials. In both cases mean ratings for all questions are well above the mid-point on the scale and indicate a high level of satisfaction with this method of map exploration.

\section{CONCLUSIONS}

The MoBIC Travel Aid is a new type of aid designed to increase the independent mobility of blind and partially sighted people. The development of the aid was based on an extensive study of user requirements and the continued involvement of users is an important feature of the project. Evaluations have been conducted with prototypical components of the device throughout the project and realistic field trials have been undertaken. Results of these studies indicate a high level of satisfaction with the MoTA. It is anticipated that a commericial version of the MoPS component will be available for both Germany and the U.K. from our industrial partner, F.H. Papenmeier GmbH, in the course of 1997, followed by later commercialization of the MoODS component.

\section{Acknowledgements}

The work described in this paper was conducted for the MoBIC Project (TP 1148) supported by the Telematics Applications for the Integration of Disabled and Elderly People (TIDE) Programme of the Commission of the European Union (DG XIII). We would like to thank our project partners and the many people who participated in the MoBIC user studies for their time and assistance. 


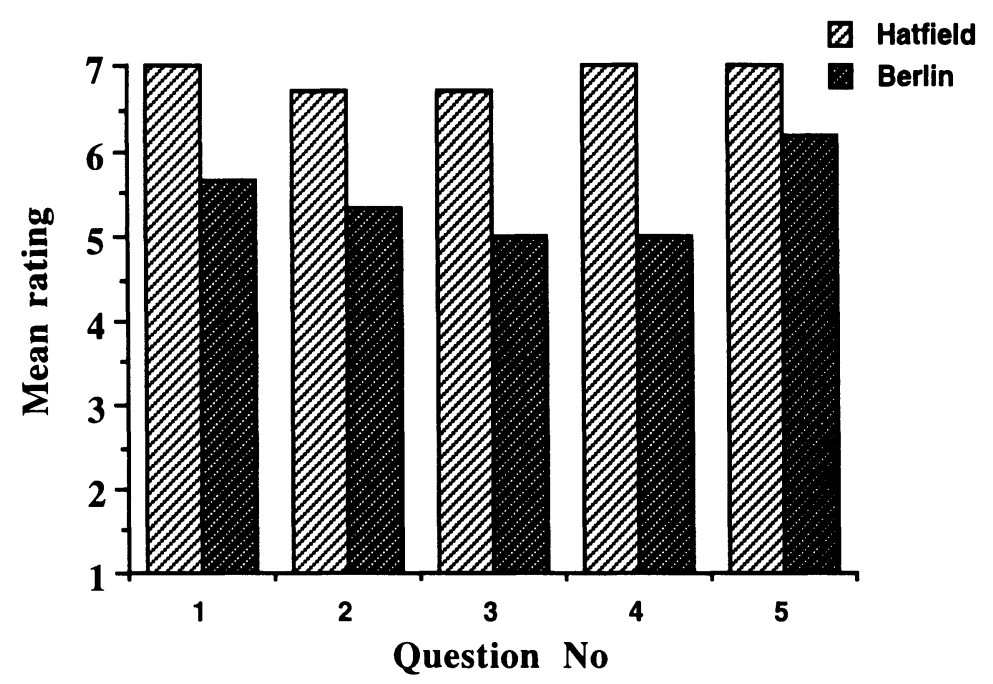

Q1 Overall how easy did you find it to operate this system?

Q2 How easy was it to learn to use this system?

Q3 How efficient did you think the system was for giving information?

Q4 How easy did you find it to build up a meaningful picture of the route exploring it in this way?

Q5 How satisfied are you with this method of obtaining map information

Figure 4: Ratings of cursor key method of map exploration in Hatfield and Berlin

\section{References}

Jansson, G. (1995). (Ed.), Requirements for effective orientation and mobility by blind people. London:

Royal National Institute for the Blind.

Landauer, T.K. (1987). Psychology as the mother of invention. Proceedings of CHI+GI '87: Conference on Human Factors in Computing Systems and Graphics Interface. New York: ACM Press.

McMillan, W.W. (1992). Computing for users with special needs and models of computer-human interaction. Proceedings of Conference on Human Factors in Computing Systems (CHI' '92). New York: ACM Press.

Mynatt, E. D., \& Weber, G. (1994). Nonvisual

presentation of graphical user interfaces: contrasting two approaches. Proceedings of Conference on Human Factors in Computing Systems (CHI '94). New York: ACM Press.

Nielsen, J. (1993). Usability engineering. Boston: Academic Press.
Norman, D. A., \& Draper, S. W. (1986). User-centered system design. Hillsdale, NJ: LEA.

Petrie, $H$. (in press). User-centred design and evaluation of adaptive and assistive technology for disabled and elderly users. Informationstechnik und Technische Informatik.

Petrie, H. and Johnson, V. (in press). MoBIC: designing a travel aid for blind and elderly people. British Journal of Visual Impairment.

Petrie, H. Morley, S., and Weber, G. (1995). Tactilebased direct manipulation in GUIs for blind users. Proceedings of Conference on Human Factors in Computing Systems (CHI '95). New York: ACM Press.

Schuler, D., \& Namioka, A. (1993). Participatory design: principles and practices. Hillsdale, NJ: LEA. Whiteside, J., Bennett, J., \& Holtzblatt, K. (1988). Usability engineering: our experience and evolution. In M. Helander (Ed.), Handbook of human computer interaction. Amsterdam: North-Holland Press. 